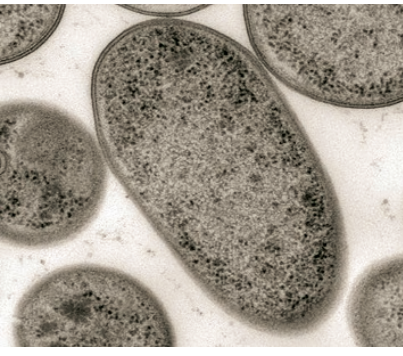

Transmission electron micrograph of $\mathrm{T}_{\mathrm{H}} 1$ cell-inducing Klebsiella pneumoniae $2 \mathrm{H} 7$. Image courtesy of S. Narushima and K. Toyooka.

GUT MICROBIOTA

\title{
Oral bacteria: a cause of IBD?
}

In a new study published in Science, oral-derived microbiota were shown to ectopically colonize the intestines of mice, eliciting a high Thelper $1\left(\mathrm{~T}_{\mathrm{H}} 1\right)$ cell response and gut inflammation. The findings suggest that intestinal pathobionts that exacerbate IBD might reside in the oral cavity.

The gut microbiota of patients with IBD are frequently enriched in oral-derived microbiota compared with that of healthy individuals; however, a causal role for these bacteria has remained undefined. Now, in the latest study, the investigators sought to identify and isolate oral bacteria that have an effect in the gut.

First, the researchers orally administered germ-free mice with saliva samples obtained from two patients with IBD. In mice that received a saliva sample from one of the two patients, a marked accumulation of IFN $\gamma^{+} \mathrm{T}_{\mathrm{H}} 1$ cells in the intestinal lamina propria was noticed.

"From mice that exhibited a high $\mathrm{T}_{\mathrm{H}} 1$ cell response in the intestine, we isolated and cultured as many microorganisms as possible, each of which was then inoculated into other germ-free mice to survey which strain was responsible," explains author Kenya Honda. These experiments identified Klebsiella pneumoniae $2 \mathrm{H} 7(\mathrm{Kp}-2 \mathrm{H} 7)$ as a strong inducer of $\mathrm{T}_{\mathrm{H}} 1$ cells when it colonizes the gut.

$\mathrm{Kp}-2 \mathrm{H} 7$ was resistant to multiple antibiotics and was found to colonize the colon and caecum when the intestinal microbiota was dysbiotic in specific-pathogen-free mice. Furthermore, in colitis-prone $\mathrm{Il} \mathrm{O}^{-/-}$mice (but not wild-type mice), $\mathrm{Kp}-2 \mathrm{H} 7$ colonization led to severe colonic inflammation, suggesting that this strain acts as a gut pathobiont in genetically susceptible hosts.

"Our results suggest the existence of a subtype of IBD, in which ectopic colonization by oral-derived bacteria in the intestine might causally relate to aberrant activation of the immune system," explains Honda. The researchers are now planning to investigate a new therapeutic strategy that specifically targets Klebsiella spp.

"Our data suggest that there are members of the normal gut microbiota that can provide colonization resistance against Klebsiella spp.," concludes Honda. "If we could identify such beneficial bacteria, we could use them as a live biotherapeutic product to treat patients with infections from multidrug-resistant Klebsiella spp."

Iain Dickson

ORIGINAL ARTICLE Atarashi, K. et al. Ectopic colonization of oral bacteria in the intestine drives

$\mathrm{T}_{\mathrm{H}} 1$ cell induction and inflammation. Science 358 , 359-365 (2017) 